Michael Schwarze (Konstanz)

\title{
Do ut des oder wie sDanter in der Commedia mit den Seelen handelt
}

Riassunto: Il quesito che fa da sfondo al presente lavoro è quello di individuare, nella Commedia, argomentazioni o procedimenti discorsivi che mostrino analogie strutturali con principi fondamentali dello scambio di denaro. L'analisi si concentra sugli scambi di battute, perlopiù brevi, fra Dante-personaggio e le anime che incontra nell'Inferno e nel Purgatorio. In questi dialoghi, Dante si serve di un modello argomentativo di stampo mercantile: infatti, essi si presentano spesso come delle trattative in cui, seguendo il principio del Do ut des, si scambiano favori immateriali. Partendo da questo assunto, il saggio propone alcune microletture che s'interrogano sul profitto di tali scambi - sia per le anime che vi partecipano, sia per l'io narrante della Commedia. Ne emergerà che, a più riprese, il modello argomentativo mercantile sovrasta il modello esegetico cristiano, che pur gli è generalmente sovraordinato. In questo modo, dal testo dantesco scaturisce una fondamentale ambivalenza: gli affari di scambi assolvono sia ad una funzione esemplare che autocostruttiva. Questa doppia strategia della Commedia, infatti, consiste nel fatto che gli scambi di favori immateriali messi in scena da essa sono sia affari conclusi nel nome di Dio che nel nome di un autore alla ricerca di autorità.

"Geist und Geld bei Dante« - das Thema stellt für Literaturwissenschaftler eine besondere Herausforderung dar. Denn um sich sachkundig zum Verhältnis von Geist und Geld bei Dante zu äußern, bedarf es, recht besehen, eingehender Fachkenntnisse auf den Gebieten der Wirtschafts- und Sozialgeschichte, der ökonomischen Theorie oder der Ordensgeschichte des italienischen Spätmittelalters. In Ermangelung entsprechender Kompetenzen wird die thematische Vorgabe »Geist und Geld" hier gewissermaßen metonymisch aufgefasst, im Sinne der grundsätzlichen Frage danach, wo Texte Dantes Motive, Argumentationen und rhetorische oder diskursive Verfahren aufweisen, die als Analogien struktureller Art zu grundlegenden Prinzipien des (Geld-)Tauschs aufgefasst werden können.

Die folgenden Überlegungen konzentrieren sich dabei auf eine spezifische Gemeinsamkeit, die den Tausch von materiellen und immateriellen Gütern gleichermaßen kennzeichnet: Gemeint ist der Nutzen, der beiden Seiten eines Handels sowohl unter Einsatz von Geld als auch beim Austausch von Handlungen 
oder Gedankengut idealiter zuwächst. ${ }^{1}$ Die Auffassung, dass Reziprozität ein gemeinsamer Nenner beim Tausch von Geist wie von Geld ist, basiert auf der Prämisse, dass materielle wie geistige Güter ein Eigentum darstellen, dessen Wechsel möglichst allen Beteiligten nutzen soll. Wenn eine Sache, ein Wissen oder eine Dienstleistung gegen Geld den Besitzer wechselt, liegt dieser beiderseitige Nutzen auf der Hand. Dort wo hingegen ein geistiges Gut gegen ein anderes weitergegeben wird, ist der beiderseitige Gewinn anders gelagert: Für denjenigen, der Gedankengut eines anderen rezipiert, besteht er, vereinfacht gesagt, nach Möglichkeit darin, dass sich sein Wissen vermehrt. Das Mehr desjenigen hingegen, der die Früchte seiner geistigen Tätigkeit darlegt, liegt darin, dass sich sein Ruf und damit potentiell sein `Marktwert`, etwa jener in der scientific community, erhöht, sofern die Vermittlung überzeugend gelingt. Ein entscheidender Unterschied zwischen dem Geldtausch und dem Gedankentausch besteht freilich darin, dass beim Geldhandel das Eigentum den Besitzer wechselt - es wird veräußert. Im Falle des geistigen Austauschs hingegen geht mit der Übertragung von Wissen deren Verbreitung einher, ohne dass der Besitz an irgendeiner Stelle weniger würde.

\section{I.}

Bricht man die Frage nach dem Verhältnis von »Geist und Geld« auf solch einfache Kategorien herunter, die den Tausch von materiellem und ideellem Besitz kennzeichnen, ergeben sich aufschlussreiche strukturelle Ähnlichkeiten zwischen ihnen und Dantes Werk. Dies gilt zumal für die Divina Commedia, denn sie thematisiert den Austausch geistiger Güter fortlaufend, wenn auch diskret. Dies geschieht nicht zuletzt in vielen Dialogfragmenten zu Beginn der Gespräche, welche die Ich-Figur mit unzähligen Seelen in den drei Jenseitsreichen führt. Besonders eingangs geht es nämlich in den Dialogen häufig darum, ob und unter welchen Bedingungen die Verstorbenen Dante an dem Wissen und den Erfahrungen teilhaben lassen, welche sie aus dem Leben mit in das jenseitige Seelendasein genommen haben. Es zeichnet sich dabei in vielen Fällen eine Art geistiger Handel ab, der nach dem Rechtsgrundsatz des Do ut des erfolgt und grundsätzlich die Frage nach dem Gewinn der Beteiligten an einem solchen Handel aufwirft. Indem Dante solche Verhandlungen punktuell immer wieder explizit inszeniert

1 Siehe in soziologischer Perspektive zum Prinzip der Reziprozität in modernen Gesellschaften Frank Adloff/Steffen Mau (Hrsg.), Vom Geben und Nehmen. Zur Soziologie der Reziprozität, Frankfurt/New York 2005 sowie Frank Hillebrandt, Praktiken des Tauschens. Zur Soziologie symbolischer Formen der Reziprozität, Wiesbaden 2009. 
oder zumindest evoziert, unterlegt er den Text der Commedia mit einem Argumentationsmuster, das sich analog zum merkantilen Handel verhält. Der semantische Kern dieses Musters besteht dabei darin, dass die Darstellung die Vergabe von Wissen an den personaggio-poeta >Dante ${ }^{2}$ vielfach als ein Tauschgeschäft ins Bild setzt, dessen Zweck in einem näher zu bestimmenden beiderseitigen geistigen Nutzen besteht.

Von Interesse ist dieser simple Textbefund, weil er im konzeptionellen Rahmen des poema sacro ein erhebliches Konfliktpotential in sich birgt. Dieses ergibt sich daraus, dass besagte Thematik des geistigen Handels in der Göttlichen Komödie in Relation zu einer übergeordneten Gesamtkonstruktion steht, die bekanntlich ihrerseits alles menschliche Handeln und alle geschichtliche Welt sub specie aeternitatis betrachtet. Die Brisanz ist zunächst moraltheologischer Art und liegt darin begründet, dass dort, wo die Erlangung des Seelenheils und schlussendlich die Gnade Gottes auf dem Spiel steht, der Nexus von Handel und Nutzen den Gedanken nahelegt, das summum bonum ließe sich mit menschlichen Mitteln verdienen. Die scholastische Gnadenlehre jedoch, auf der Dantes Jenseitskonstruktion fußt, schließt einen solchen Verdienstgedanken kategorisch aus: Im Gegenteil, denn ihr zufolge ist die grazia Dei ein »grundloses Geschenk» (»dono gratuito«), das ausschließlich aus dem unergründlichen Willen Gottes erwächst. ${ }^{3}$ Der grundlegende Beitrag, den der Mensch zur Erlangung dieser Gnade leisten kann und muss, bestand nach den Lehren von Pietro Lombardo, dem Heiligen Bonaventura und Thomas von Aquin in der grundsätzlichen Bereitschaft, dem Willen des Schöpfergottes zu folgen. Bei dieser Wahlfreiheit handelt es sich nach der scholastischen Lehre um eine den Christenmenschen von Natur aus gegebene Disposition und nicht etwa um eine Freiheit, die sich in einzelnen Handlungsentscheidungen manifestiert. Die Erlangung der göttlichen Gnade aufgrund individueller Fähigkeiten, Eignungen oder Verdienste schließt die zeitgenössische Kirchenlehre auf diese Weise per definitionem aus. Dies bedeutet, dass Vorstellungen einer >Gnadenökonomieく vom theologischen Standpunkt aus streng genommen widersinnig sind.

2 Siehe zu den Rollen der Dante-Figur in der Commedia und ihrer doppelten Lesbarkeit Gianfranco Contini, »Dante come personaggio-poeta della `Commedia« ( $\left.{ }^{1} 1958\right)$, zuletzt in: ders., Un’idea di Dante, Turin 1976, S. 33-62. Um die Dante-Figur im Text vom empirischen Dante Alighieri zu unterscheiden, wird der Name der ersten im Folgenden in einfache Anführungsstriche gesetzt.

3 Siehe hierzu sowie zu Grundzügen der theologischen Fundierung der grazia Dei bei Dante konzise Andrea Ciotti, »La nozione di grazia divina in Dante«, in: Enciclopedia Dantesca, a cura di Umberto Bosco, Istituto della Enciclopedia Italiana fondata da Giovanni Treccani, Roma ${ }^{2} 1971$, Bd. III, S. 274-277. 
Anders sieht es hingegen aus, wenn man bedenkt, dass ein solches Gedankenkonstrukt in der Commedia zugleich im Kontext eines dichterischen Entwurfs des christlichen Jenseits steht: Der poema handelt im Kern von der Geschichte eines in Sünde gefallenen Dichters, der von seiner Suche nach Manifestationen des göttlichen Erlösungswerkes in den Reichen der Toten erzählt. Im Rahmen dieser narrativen Konstellation, so die These der folgenden Überlegungen, begründet die Überlagerung der beiden angesprochenen gedanklichen Ordnungen ein latentes Konkurrenzverhältnis, dem ein ambiguisierendes Sinnpotential innewohnt. Letzteres besteht darin, dass in der Gleichzeitigkeit von dominierend christlicher Heilslehre und punktuell aufgerufener merkantiler Motivik eine grundlegende Ambivalenz erkennbar wird. Es handelt sich dabei um eine Strategie des Textes, die gezielt diskrepanten Lesarten der Commedia den Weg bahnt.

\section{II.}

Um dies zu zeigen, gilt es als Erstes dem Befund eines Argumentationsmusters, das die Rechtsformel des do ut des variiert, textuell Substanz zu verleihen. Es ist auf thematischer Ebene angesiedelt und ergibt sich aus der Mikroanalyse der vielen Informationsgespräche, die das Ich in den ersten beiden Cantiche mit den von ihren irdischen Sünden und Lastern geplagten Seelen führt. In diesen Dialogen, deren strukturelle Bedeutung in der Danteforschung meines Wissens bisher keine eingehende Beachtung gefunden hat, geht es >Dante< zunächst stets darum, den Namen seines jeweiligen Gegenübers zu hören. Darüber hinaus versucht er die Gründe in Erfahrung zu bringen, die dazu geführt haben, dass deroder diejenige nach dem Tode gemäß der kosmologischen Ordnung dorthin transferiert wurde, wo ১Dante` auf ihn oder sie trifft. In persuasiver Absicht schlägt er seinen potentiellen Informanten dabei in meist sehr kurzen Gesprächssequenzen eine Art geistigen Handel vor.

So auch am Ende von Inferno XXXII, als er auf dem tief gefrorenen See Kozytus auf die Seele des namentlich noch nicht erwähnten Pisaner Grafen Ugolino trifft. ${ }^{4}$ Als >Dante` sieht, wie dieser Mann von Hass erfüllt seine Zähne roh in den Nacken einer unter ihm befindlichen Gestalt rammt, schlägt er ihm unvermittelt eine Art Geschäft vor. Es heißt dort:

4 Siehe grundlegend zu dem Gesang Anna Maria Chiavacci Leonardi, »Il canto disumano«, in: L'Alighieri 25, 1 (1984), S. 23-46. 
»O tu che mostri per sì bestial segno

odio sovra colui che tu ti mangi,

dimmi 'l perchè [...] per tal convegno,

che se tu a ragion di lui ti piangi,

sappiendo chi voi siete e la sua pecca,

nel mondo suso ancora io te ne cangi, [...] (Inf. XXXII, 133-138)

Signifikant ist diese Sequenz zum einen, weil sie der Ich-Figur explizit das Substantiv »convegno« (V. 135) in den Mund legt, was so viel wie >Absprache oder ,Übereinkunft ‘ bedeutet. Der Passus markiert damit lexikalisch, dass der Handel, den `Dante` dem anderen anträgt, auf gegenseitigem Einverständnis beruhen soll. Letzteres gibt ihm Ugolino stillschweigend, indem er im folgenden 33. Gesang ausführlich vom Vertrauensbruch des Erzbischofs Ruggieri degli Ubaldini und dem tragischen Hungertod erzählt, den seine Söhne und Ugolino selbst erlitten. ${ }^{5}$ Zum anderen benennt die Sequenz beispielhaft das Prinzip des beiderseitigen Nutzens, von dem eingangs die Rede war. Im vorliegenden Fall, so erklärt Dante, solle Ugolino ihn umfassend darüber unterrichten, wer er und der andere seien, warum er den anderen vor Hass buchstäblich auffresse und schließlich, welcher Sünde jener sich schuldig gemacht habe. Im Gegenzug, so stellt er für den Fall, dass Ugolino den Bischof zu Recht anklagt, in Aussicht, werde er es ihm »oben in der Welt« nach seiner Rückkehr vergelten. Was mit der verheißungsvollen Formulierung "nel mondo suso ancora io te ne cangi« (V. 138) gemeint ist, womit also das Ich der Rede Ugolinos Bericht konkret zu vergelten gedenkt, bleibt an dieser Stelle offen. Einen vagen Hinweis darauf gibt lediglich die Bedingung, die >Dantes für das erfolgreiche Zustandekommen des Handels stellt: »se tu a ragion di lui ti piangi« (V. 136, Hervorhebung M.S.). Der Konditionalsatz bringt die Möglichkeit zum Ausdruck, dass sich Ugolino zu Recht oder zu Unrecht beklagt, und macht seine Aussagen somit gewissermaßen zum Gegenstand eines Rechtsverfahrens. Die Instanz aber, die in diesem Verfahren offensichtlich stillschweigend das Richteramt für sich beansprucht, ist kein anderer als Dantes Ich in seiner Rolle als Dichter und Verfasser der Commedia. >Dante` attribuiert sich damit recht unverbrämt die souveräne Rolle eines zweiten Richters, der das Urteil des Jüngsten Gerichts gleichsam einer Revision unterziehen, sein Urteil fällen und den göttlichen Richterspruch entsprechend sanktionieren wird. ${ }^{6}$ Ohne dass wir dadurch

5 Siehe dazu Inf. XXXIII, 1-75. In diesem Kontext wäre eine nähere Betrachtung der Verse 16-18 lohnend, da sie davon berichten, wie Ruggeri eine Abmachung brach, die auf Vertrauen beruhte.

6 Siehe zu dieser unerhörten Rolle, die sich das Ich der Commedia implizit zuspricht, Joachim Küpper, »Bilder der Sünde: Dante und Botticelli«, in: Deutsches Dante-Jahrbuch 81 (2006), S. 155173. Küpper konstatiert, das höchstrichterliche Urteil des Jüngsten Gerichts werde in vielen Begegnungen >Dantes` mit den Seelen durch die Augenblickhaftigkeit der emotionalisierten 
bereits wüssten, worin die Sanktionsinstrumente des personaggio poeta bestehen, lässt sich bis hierher festhalten, dass die Vereinbarung zwischen >Dante und Ugolino implizit als ein Geschäft zwischen Ungleichen ausgewiesen wird. Diskursiv wird diese Asymmetrie zusätzlich dadurch unterstrichen, dass Dante die Abmachung gewissermaßen einseitig verkündet und Ugolino die ihm zugewiesene inferiore Position wortlos und, wie es den Anschein hat, selbstverständlich akzeptiert. Es zeichnet sich hier eine Hierarchie zwischen den Beteiligten des Handels ab, die für das in Frage stehende Argumentationsmuster symptomatisch ist. Denn hinter der Überordnung der Ich-Figur über Ugolino und viele andere jenseitige Figuren steht eine asymmetrische Handlungslogik. Diese manifestiert sich in der Art der Gegenleistung, die >Dante für die Informationen, mit denen seine Handelspartner in Vorlage treten, erbringt: Sein Anteil besteht darin, dass der Dichter die Namen der Seelen und ihre Schicksale in das Who is who seiner Commedia aufnimmt. Auf diese Weise, so wird dem Leser vermittelt, verschafft er den Verstorbenen durch seine Dichtung ein Nachleben auf Erden und mehrt gegebenenfalls ihren Ruhm. ${ }^{7}$ Diese Fiktion setzt voraus, dass das Urteil über die Sünden der Verstorbenen in der diesseitigen Welt im Gegensatz zur jenseitigen noch nicht endgültig gefällt ist, sondern parteiischen Wertungen unterliegt, die sich wandeln können. Für die jenseitigen Sünder, die naturgemäß nicht freiwillig über sich reden wollen, da sie nur Schändliches zu berichten haben, bedeutet dies eine Verlockung. Sie besteht in der Möglichkeit, dass ihre zu Lebzeiten begangenen Vergehen im Lichte von >Dantes` Nachruf im Urteil zukünftiger Generationen $\mathrm{zu}$ verzeihlichen oder gar guten Taten werden und sie auf diese Weise, selbst wenn sie schlecht beleumundet bleiben, zumindest nicht dem Vergessen anheimfallen. Getrieben von dieser Hoffnung verhandeln sie mit >Dante über die Bedingungen ihrer >Aussage ${ }^{8}{ }^{8}$ In diesem Sinne inszeniert der Text Tauschgeschäfte, bei denen >Dante`, der Dichter von Rang, in der Funktion eines vertrauenswürdigen Bürgen auftritt, der einen moralisch verlässlichen Nachruf der Seelen gewährleisten kann.

Reaktionen des Ich insgeheim einer individuellen Prüfung unterzogen. So jedoch rolle die Commedia die >Verfahren` des Jüngsten Gerichts vor den Augen des Lesers gewissermaßen neu auf.

7 Auf diese Weise verleiht der Text der Commedia vielen Gestalten überhaupt erst einen Namen und macht sie identifizierbar. Auf den prekären Status, den dieses Prinzip der »nominanza« im Kontext der superbia-Thematik für die Ich-Rede bedeutet, hat überzeugend Teodolinda Barolini hingewiesen: The Undivine Comedy. Detheologizing Dante, Princeton 1992, S. 132-142.

8 Der ungewisse Leumund auf Erden ist aus Sicht der Seelen der wesentliche Grund für ihre Verhandlungen mit >Danteく. Dies unterscheidet sie m.E. grundlegend vom Angeklagten vor Gericht, der bereits vorab weiß, dass eine ehrliche Darstellung seiner Vergehen ihm nützen wird. 
Eine Textstelle, die diesen Konnex in seltener Klarheit thematisiert, findet sich erneut im 32. Gesang des Inferno, dessen einzigartige Darstellung offener Konfliktualität für unseren Zusammenhang ausgesprochen aufschlussreich ist. In der Mitte des Canto kommt es zu dem handgreiflichen Zusammenstoß >Dantes` mit dem politischen Verräter Bocca degli Alberti. Nachdem er beim Gang durch die im Eis festgefrorenen Körper grob gegen dessen Gesicht gestoßen ist und Bocca ihn daraufhin äußerst aggressiv angefahren hat, was er überhaupt in Antenora verloren habe, macht Dante folgenden Vorschlag zur Güte:

»Vivo son io, e caro esser ti pote»

fu mia risposta, »se dimandi fama,

ch'io metta il nome tuo tra l'altre note.« (Inf. XXXII, 91-93)

>Dante gibt sich hier gewohnt selbstbewusst als Dichter aus, der grundsätzlich bereit ist, den Namen Boccas in seine »note« aufzunehmen. Mit ihnen können dem Stellenkommentar von Chiavacci Leonardi zufolge nur die Aufzeichnungen, die zur Abfassung der Commedia gedient haben, gemeint sein. ${ }^{9}$ Explizit verbindet der Dichter damit die Aussicht, Bocca könne dadurch die allseits erstrebte »fama« (V. 92) in der Nachwelt erlangen, welcher er selbst als Lebender angehört. Als Bocca freilich nicht daran denkt, auf Dantes Vorschlag einzugehen, seinen Namen also nicht preisgibt ${ }^{10}$ und die Situation eskaliert, verbietet der Dichter ihm gleichsam das Wort und droht ihm nun, zu seiner "Schande« »wahre Nachrichten « über ihn zu verbreiten. Der Ton, in dem er dies tut, erinnert an einen Richterspruch, der in letzter Instanz ein Urteil über eine Person verkündet:

\footnotetext{
»Omai«, diss'io »non vo' che tu favelle, malvagio traditor, ch'alla tua onta io porterò di te vere novelle.« (Inf. XXXII, 109-111)
}

Entscheidend für unseren Zusammenhang ist an dem Passus, dass >Dante seinem Gegenüber bei Wohlverhalten Ruhm oder wenigstens einen guten Ruf verheißt, während das Missverhalten Boccas zur Konsequenz hat, dass der Dichter ihn als »boshaften Verräter« (V. 110) darstellen wird. Auf diese Weise vermittelt die Szene, das literarische Zeugnis der Commedia könne solches Ansehen garan-

9 Vgl. Dante Alighieri, Commedia, con il commento di Anna Maria Chiavacci Leonardi, vol. 1, Inferno, Milano 1991, S. 962.

10 Die Frage des nominarsi (vgl. V. 93, V. 98) erscheint dabei in >Dantes Worten als der entscheidende Punkt, denn erst als der Dichter diesen aus dem Mund eines Dritten erfährt (V. 106), lässt er von Bocca ab. 
tieren oder eben verhindern. Zugleich signalisiert die Sequenz damit eine Gleichsetzung der »note« des Dichters mit der Möglichkeit, für die in ihnen genannten Personen in den Genuss posthumer »fama» zu gelangen. Mit »fama« ist im Kontext der Do-ut-des-Geschäfte dabei in der Regel offensichtlich Bekanntheit auf Erden gemeint. Dies verdeutlicht prägnant eine Stelle am Ende von Purgatorio XIII, in der die entgegen ihrem Namen zu Lebzeiten törichte Sapia de' Salvani >Dante ‘ im Gespräch bittet, ihr bei ihren noch lebenden toskanischen Verwandten erneut zu gutem Ansehen zu verhelfen. ${ }^{11}$ Nachdem die Sieneserin dem wissbegierigen >Dante` aus freien Stücken erzählt hat, was sie auf den Läuterungsberg geführt hat und er ihren »spirito eletto« (V. 142) im Gegenzug aufgefordert hat, sie um etwas zu bitten, was er »drüben« mit seinen »sterblichen Füßen« für sie erledigen könne, antwortet sie:

»E cheggioti, per quel che tu più brami, se mai calchi la terra di Toscana, che a'miei propinqui tu ben mi rinfami.«(Purg. XIII, 148-150) ${ }^{12}$

Von solch einem tatkräftigen Eintreten heißt es - erneut in Inf. XXXII -, es könne Bocca »caro« (V. 91), »lieb« sein. Die Finalität, die auf diese Weise in dem Höllengesang zwischen den Aufzeichnungen und dem zu erwartenden Ruf auf Erden hergestellt wird, supponiert für den Autor der Commedia insgeheim ein Prestige, auf dessen Bedeutung im letzten Abschnitt dieser Überlegungen zurückzukommen sein wird.

Zuvor jedoch gilt es den Textbefund mit Blick auf das Purgatorium und das Paradies zu erweitern. Auf dem Läuterungsberg bietet das Ich in den Tauschgeschäften mit den von ihren ehemaligen Lastern geschundenen Seelen noch eine zusätzliche Gegenleistung für die Informationen, die sie ihm liefern, an. Diese besteht in Fürbitten und Gebeten, mit denen nach kirchlicher Lehre die Lebenden dazu beitragen können, dass die Seelen der Verstorbenen das Reich Gottes schneller erlangen. Von solchen »buon preghi«, durch die man »von dort - gemeint ist das Purgatorio - sehr gut vorankommt«, ist in der zweiten

11 Siehe grundlegend zu Purg. XIII Andreas Kablitz, »Videre - Invidere. Die Phänomenologie der Wahrnehmung und die Ontologie des Purgatoriums. (Dante, Divina Commedia, Purgatorio XIII)«, in: Deutsches Dante-Jahrbuch LXXIV (1999), S. 137-188. Zur Figur der Sapia Giuliana Angiolillo, „Sapia de’ Salvani. Canto XIII«, in: dies., La nuova frontiera della tanatologia: Purgatorio, Firenze (Olschki) 1996, S. 131-145.

12 Bei dem Verb »rinfamar« handelt es sich nach Chiavacci Leonardi um eine Wortschöpfung Dantes: vgl. Dante Alighieri, Commedia, con il commento di Anna Maria Chiavacci Leonardi, Bd. II, Purgatorio, Milano 1994, S. 400. 
Cantica häufig die Rede, vor allem in Bezug auf zahlreiche Personen der Zeitgeschichte. ${ }^{13}$ Ein Beispiel, in dem das uns interessierende Argumentationsmuster dabei mit seltener Klarheit zum Ausdruck kommt, findet sich in Purg. XXVI, als dem Jenseitspilger aus dem reinigenden Feuer der purgatio die Minnedichter Guido Guinizelli und Arnaut Daniel entgegenkommen, freilich in einer Gestalt, die sie zunächst unkenntlich macht. Nachdem >Dante` ihnen erläutert hat, wieso es ihm vergönnt ist, seinen »sterblichen Teil« durch ihr Reich zu bringen, fordert er die beiden Dichtervorfahren auf, sich selbst zu erkennen zu geben und ihn über eine andere Gruppe von Seelen, die sich schemenhaft nähert, aufzuklären. Auch in diesem Dialog verbindet der Dichter mit seiner Bitte um Informationen das Versprechen, mit diesen später die Blätter seiner Commedia zu füllen (vgl. V. 64). Der wahre Lohn aber, der ihnen dadurch ausdrücklich verheißen wird, besteht, salopp formuliert, in einer Beschleunigung ihres Aufnahmeverfahrens in das Paradies. Unter der Voraussetzung nämlich, dass die Unerkannten ihm die gewünschten Informationen geben, werde ihr größter Wunsch bald gestillt und sie würden in den Himmel aufgenommen:

\author{
Ma se la vostra maggior voglia sazia \\ tosto divegna, sì che 'l ciel v'alberghi \\ ch'è pien d'amore e più ampio si spazia, \\ ditemi, acciò ch'ancor carte ne verghi, \\ chi siete voi, e chi è quella turba \\ che se ne va di retro a' vostri terghi. (Purg. XXVI, 61-66)
}

Versteht man die Konjunktion in Vers 61 mit Chiavacci Leonardi als »se augurativo« mit finalem Sinn, ${ }^{14}$ bedeutet dies, dass den Berichten, die das Ich über seine jenseitigen Begegnungen in dem poema sacro niederschreibt, hier gleichsam eine karitative Funktion zugesprochen wird. Denn >Dantes` Angebot besagt nichts anderes, als dass seine Commedia einen entscheidenden Beitrag zur Aufnahme der berühmten Dichtervorfahren in die civitas Dei leisten kann und wird, sofern

13 Die Zitate sind Purgatorio III entnommen, das den eigentlichen Handlungsbeginn der zweiten Cantica markiert und in dessen Zentrum die bußfertige Rede des Stauferkönigs Manfred steht. Die fundamentale Gültigkeit des Prinzips einer Verkürzung der purgatio durch die karitative Fürbitte der Lebenden wird in dem Canto dadurch exponiert, dass der Staufer als Abschluss seiner vergleichsweise langen Rede gewissermaßen ex catedra erklärt: »Vero è che quale in contumacia more / di Santa Chiesa, ancor ch'al fin si penta, / star li convien da questa ripa in fore, / per ognun tempo ch'elli è stato, trenta, / in sua presunzïon, se tal decreto / più corto per buon prieghi non diventa. / Vedi oggimai se tu mi puoi far lieto, / revelando a la mia buona Costanza / come m' hai visto, e anco esto divieto; / ché qui per quei di là molto s'avanza« (Purg. III, 136-145).

14 Vgl. Chiavacci Leonardi (wie Anm. 12), S. 774. 
sie einwilligen. Damit aber steht die Passage in argumentativer Analogie zu dem Vaterunser, um das Guido Guinizelli den >privilegierten`Menschen >Danteく wenig später genau zu demselben Zweck ausdrücklich bittet. ${ }^{15}$ Eine solch moraltheologisch begründete Variante unseres Motivkomplexes leuchtet im Kontext des Purgatorio unmittelbar ein, ist dieses doch ein jenseitiger Raum, welcher in der ultramundanen Rechtsordnung der Commedia gleichsam als Bewährungsraum der büßenden Seelen fungiert und dem insofern im Kontrast zur temporalen Stasis der Hölle eine zeitliche Dimension zu eigen ist.

Im Paradiso spielt eine derartige Semantisierung des geistigen Handels eine geringere Rolle. Dies ist kaum überraschend, denn dort, wo die Seelen das Gottesheil bereits erlangt haben, brauchen und können der Christenmensch >Dante und seine Dichtung nicht mehr entscheidend für sie eintreten. Gleichwohl findet das Argumentationsmuster auch in dieser Cantica vereinzelt Anwendung. Mit dem Unterschied allerdings, dass das Dichter-Ich in der Hierarchisierung der Dialogpartner gewissermaßen die Rolle tauscht: Denn aus dem in seiner Mission gegenüber den Seelen äußerst selbstbewusst auftretenden >Dante des Inferno und des Purgatorio wird im Paradiso ein Bittsteller. Im Reich der Erlösten tritt er in demütiger Pose auf und erfleht von der »gloria di colui che tutto move« (Par. I, 1) vom ersten Vers an vor allem eines: dass ihm, dem Dichter, die Gabe verliehen werde, das Unbegreifliche, das er dort erlebt und das die Erinnerung nicht fassen kann, gleichwohl sprachlich zu evozieren und der Nachwelt vermitteln zu können. ${ }^{16}$ Im Gegenzug gelobt er seine Dichtung voll und ganz in den Dienst der Verkündigung eben jener grazia Dei zu stellen, derer er im Paradies teilhaftig geworden ist. Besonders prägnant bringt den Nexus zwischen den in letzter Instanz von Gott erbetenen dichterischen Fähigkeiten und der korrespondierenden Verpflichtung >Dantes`, das Lob seines Schöpfergottes zu singen, der 33. und letzte Gesang der Commedia zum Ausdruck. Epilogisch ruft das Ich in ihm in einer Weise das »höchste Licht« an, die Hartmut Köhler dazu veranlasst hat, ausdrück-

15 »Or se tu hai sì ampio privilegio, / che licito ti sia l'andare al chiostro / nel quale è Cristo abate del collegio, / falli per me un dir d'un paternostro, / quanto bisogna a noi di questo mondo, / dove poter peccar non è più nostro«. (Purg. XXVI, 127-132).

16 »Nel ciel che più de la sua luce prende / fu' io, e vidi cose che ridire / né sa né può chi di là sù discende; / perché appressando sé al suo disire, / nostro intelletto si profonda tanto, / che dietro la memoria non può ire.« (Par. I, 4-9). Siehe zur Bedeutung der die gesamte Cantica umspannenden Suche nach einer geeigneten Dichtungssprache für die Thematisierung des Unbegreiflichen und dem damit einhergehenden Konzept des »trasumanar« Bodo Guthmüller, »Trasumanar significar per verba / non si poria. Zu Dantes erstem Gesang des Paradiso«, in: Deutsches DanteJahrbuch 82 (2007), S. 67-85. 
lich zu konstatieren, die »Fiktion« nehme »hier geradezu Züge eines >Do ut des` mit der Gottheit an $:^{17}$

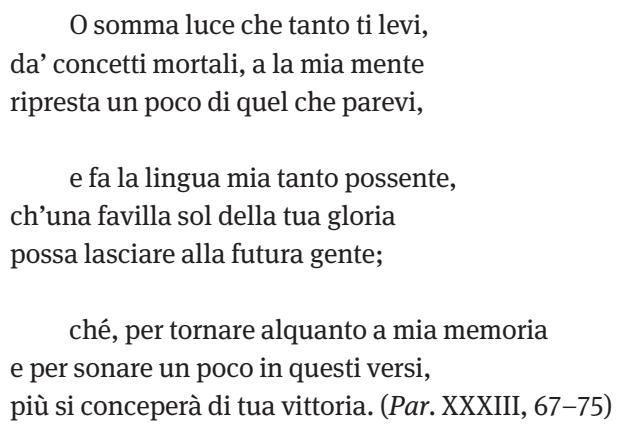

Die drei Terzinen entwerfen das Bild eines >Dante`, dem im Paradies die unbegreifliche Herrlichkeit Gottes gewissermaßen im Namen zukünftiger Menschheitsgeschlechter (»futura gente«, V. 72) erschienen ist (»parevi«, V. 69) und der seine Aufgabe darin sieht, jenen mittels des Klangs seiner »versi« (V. 74) wenigstens einen Funken (V. 71) der göttlichen Allmacht (vgl. »gloria«, V. 71) erfahrbar zu machen. Dieser Auftrag jedoch wird hier, am Schluss der Cantica, explizit an die Bedingung geknüpft, dass dem Ich seinerseits das einzigartige poetische Vermögen zuteil werden möge, das Geschaute in eine wirkungsmächtige (»possente«, V. 70) Sprache zu transponieren, welche die "gloria« Gottes und seine ewige »vittoria« (V. 75), in adäquater Form zu besingen weiß. Selbstredend muss dieser unilateral verkündete Tauschhandel zwischen dem Dichter und seinem Schöpfer an dieser Stelle offen bleiben; die »somma luce« antwortet nicht. Es fehlt der Sequenz damit das dialogische Moment des »convegno« (Inf. XXXII, 135), welches wir oben als konstitutiv für das merkantile Argumentationsmuster gekennzeichnet haben. Gemeinsam ist allen Beispielen jedoch das Prinzip des do-ut-des. Im vorliegenden Fall bringt es abschließend ein Selbstverständnis des Textes zum Ausdruck, das die Schau des summum bonum und die dichterische Meisterschaft in ein wechselseitiges Begründungsverhältnis setzt und beide Ziele mit einer vergleichbaren Exklusivität ausstattet. Dies aber bedeutet nicht weniger, als dass der demütige Gestus, mit dem >Dante $\mathrm{im}$ Paradiso den Figurationen des Prinzips göttlicher Liebe begegnet, zugleich immer auch impliziter Ausdruck seines Anspruchs auf höchste dichterische

17 Dante Alighieri, La Commedia - Die Göttliche Komödie III, Paradiso/Paradies, Italienisch/ Deutsch, in Prosa übersetzt und kommentiert von Hartmut Köhler, Stuttgart (Reclam) 2012, S. 723, Anmerkung 75. 
Könnerschaft im Diesseits ist. ${ }^{18}$ In den ersten beiden Cantiche hingegen inszeniert Dante, wie wir sehen konnten, immer wieder geistige Geschäfte, die mehr oder weniger ausgesprochen stets auf dem Prinzip des Tausches von persönlichen Informationen gegen wohlwollende Nachrede in der Commedia beruhen. Der Nutzen auf Seiten der Seelen besteht dabei dem Text zufolge darin, dass der poeta mit seiner Dichtung der Möglichkeit nach einerseits für eine gerechte "fama« der Verstorbenen unter seinen Zeitgenossen bürgt, andererseits für die baldige Erlangung des Seelenheils seiner Gesprächspartner im Purgatorio einsteht.

\section{III.}

Dem Grundsatz der Reziprozität des Tauschhandels entsprechend stellt sich die Frage nach dem Gewinn jedoch nicht nur für die Seelen der Verstorbenen, sondern genauso für `Dante`, der die Übereinkünfte in der Regel initiiert. Nicht umsonst hat der Autor Dante das >Ich der Rede als die uneingeschränkt dominierende Wahrnehmungs- und Handlungsinstanz sowie als das Organisationszentrum der Commedia angelegt. Seinen Nutzen an den Tauschgeschäften freilich thematisiert der Text kaum einmal explizit. Den Lesern wird auf diese Weise in den ersten beiden Cantiche latent insinuiert, die Figur >Dante handle gewissermaßen altruistisch und zum ausschließlichen Wohl derjenigen, die für ihre Sünden auf ewig in die Hölle verdammt sind oder auf dem Läuterungsberg für ihre lasterhaften Verfehlungen büßen. Dass dem aber schon vordergründig nicht so ist, ergibt sich aus dem bereits Ausgeführten deutlich: Denn die fortlaufenden Dialoge, in denen Dante die Seelen dazu bringt, sich zu offenbaren, inszenieren zugleich stets, wie der Dichter an den für seine Darstellung notwendigen Stoff gelangt. Der Text gibt dabei vor, die großen Mühen und das Geschick zu dokumentieren, mit denen `Dante〈 das profane Material für sein christliches Lehrgedicht gesammelt hat. Ihm gelingt es so, die

18 Siehe dazu Gerhard Regn, »Double Authorship: Prophetic and Poetic Inspiration in Dante's Paradise«, in: Modern Language Notes 122,1 (2007), S. 167-185 sowie ders., »Gott als Dichter: Die Wirklichkeit der Fiktion in Dantes Paradiso«, in: U. Peters/R. Warning (Hrsg.), Fiktion und Fiktionalität in den Literaturen des Mittelalters, München 2009, S. 365-385. Regn spitzt die hier gemachten Textbeobachtungen mit pertinenten Argumenten pointiert im Sinne einer dichterischen Legitimationsstrategie zu. So betreibe die dritte Cantica »die Stilisierung Gottes zu einem Autor, der nach den Grundsätzen weltlicher Poetik« dichte. Dante verfolge mit dieser Konstruktion nicht zuletzt »eine legitimatorische Funktion. Gleichsam unter dem Schirm göttlicher Autorisierung erlaubt sie die Aufwertung mundaner Poesie im Kontext religiöser Prophetie.« (»Gott als Dichter«, S. 376). 
Instanz seines Verfassers und das Projekt der Commedia diskret permanent präsent zu halten. Die stolze Selbstthematisierung erscheint in dieser Perspektive als offensichtlicher Gewinn, den Dante aus der Inszenierung der Tauschgeschäfte seines fiktionalen Ichs mit den Seelen zieht.

Diese Beobachtung wäre nicht weiterer Rede wert, stünde Dante, indem er sich und seine Belange in der Commedia fortgesetzt thematisiert, nicht in der Gefahr, in Konflikt mit seiner eigenen gedanklichen Ordnung zu geraten. Dieser Verdacht gründet auf der Tatsache, dass unser Autor das »Reden über sich selbst « im ersten Buch des Convivio grundsätzlich streng verurteilt und seine Zulässigkeit strengen Restriktionen unterstellt. ${ }^{19}$ Als Grund für die grundsätzliche Verwerflichkeit des "parlare di sé« nennt er dort, dass eine unvermeidbare Begleiterscheinung der Selbstrede das Selbstlob oder der Selbsttadel seien. Denn es gebe »keinen Menschen, der ein wahrer und gerechter Bewerter seiner selbst« sei. Auf diese Weise aber werde derjenige, der von sich spreche, Opfer seiner Eigenliebe und urteile sich selbst gegenüber wie der »falsche Händler«, der mit »einem Maß verkaufe und mit einem anderen kaufe «. ${ }^{20}$ Der grundlegende Einwand, den Dante gegen das Reden über sich selbst formuliert, besagt im Kern, dass kein Mensch von der Eigenliebe (»propria caritate«) absehen kann und daher stets einer verzerrenden, von aller Wahrheit abweichenden Selbsttäuschung unterliegt. Selbstlob und Selbsttadel erscheinen demnach in seinen Augen als eine ebenso unvermeidbare wie unverzeihliche Begleiterscheinung jeglicher Ich-Rede. Vor der Folie dieser Argumentation des Convivio wird deutlicher erkennbar, was Dante in der Commedia mit der vermeintlich beiläufigen Thematisierung seiner Do-ut-des-Geschäfte mit den Seelen bezweckt: Sie ermöglichen es ihm, in verkappter Form jene lobende Selbstrede zu führen, deren moralisch-ethische Fragwürdigkeit das Convivio unter Hinweis auf die Fallstricke der Eigenliebe grundsätzlich anprangert. In diesem Lichte betrachtet, erweisen sich die Vereinbarungen, die `Dante « mit den Bewohnern des oltre mondo trifft, nicht zuletzt als eine diskrete Textstrategie zur Ermöglichung einer an sich prekären Ich-Rede. Doch wieso bedient sich die Darstellung solch

19 Mit der Sigle CV zitiert nach Dante Alighieri, Das Gastmahl. Erstes Buch, Italienisch/Deutsch, übersetzt von Thomas Ricklin, eingeleitet und kommentiert von F. Chevenal, Hamburg 1996. Dantes aus philosophiegeschichtlicher Perspektive hybride Anlage des Convivio und die Folgen, die dies für die in dem Traktat angeführten Bedingungen und Möglichkeiten der Selbstaussprache hat, untersuchte eine grundlegende Studie von Andreas Kablitz, »Dantes poetisches Selbstverständnis (Convivio - Commedia)«, in: Winfried Wehle (Hrsg.), Über die Schwierigkeiten (s)ich zu sagen, Frankfurt a.M. 2000, S. 17-57; für das Folgende insbesondere S. 19-40.

20 Im Original heißt es dazu: »E ancora la propria loda e lo proprio biasimo è da fuggire per una ragione igualmente, sì come falsa testimonianza fare: però che non è uomo che sia di sé vero e giusto misuratore, tanto la propria caritate ne 'nganna. Onde aviene che ciascuno ha nel suo giudicio le misure del falso mercatante, che vende coll'una e compera coll'altra; [...]« (CV, I, ii 8-9). 
einer in konzeptioneller Hinsicht fragwürdigen Strategie? Weist die stilisierende Selbstinszenierung >Dantes` mittels der Do-ut-des-Geschäfte insgeheim eine Legitimität auf, die mit dem Gedanken des Convivio vereinbar ist? Oder negiert die Commedia in diesem Punkt schlichtweg die theoretische Reflexion Dantes und setzt ihr mit der Figur >Dantes` einen Protagonisten entgegen, dessen Grad an autonomer Selbstvergewisserung bereits in die Neuzeit weist? Im Folgenden wird zu zeigen sein, dass eine solche alternative Hypothesenbildung nicht vereindeutigend zugunsten einer der beiden Positionen entschieden werden kann, sondern ihre eigentliche Bedeutung erst erlangt, wenn man sie als einen Gegensatzzusammenhang begreift, dessen Anteile sich zueinander komplementär verhalten. Einen Hinweis darauf, dass die Selbstrede unter gewissen Umständen tatsächlich legitim sein kann, liefert die bereits zitierte Passage des Convivio, die von der Verwerflichkeit des »parlare di sé« handelt. Dante erklärt dort, es gebe durchaus Gründe, die das »Reden über sich « rechtfertigten. Ein Umstand sei, wenn man sich gegen große Verleumdungen oder Gefahren nicht anders zur Wehr setzen könne. ${ }^{21}$ Der andere sei gegeben, wenn dem »ragionare di sé« großer Nutzen dadurch erwachse, dass anderen eine »Belehrung« zuteilwerde. ${ }^{22}$ Dieser zweite Grund ist für uns von Interesse. Denn die Option einer übergeordneten »dottrina«, welche die Ich-Rede positiv sanktioniert, wirft die Frage auf, wie in dieser Hinsicht die Tauschgeschäfte zu beurteilen sind. Haben sie die Vermittlung von Informationen und die Behandlung von Gegenständen zur Folge, die in einer Weise lehrreich sind, dass sie die fortgesetzte Inszenierung des Dichter-Ich legitimieren? Hinter dieser Frage verbirgt sich jene nach etwaigen übergeordneten Begründungen, welche die insistente Darstellung eines unbändigen Wissensdursts, den `Dante ‘ in diesen Gesprächen an den Tag legt, rechtfertigen können.

Diesem Punkt nachzugehen ist opportun, war Dante bekanntlich der Gedanke vertraut, ein unmäßiger Wissensdrang sei sündhaft. Aufschlussreich ist in diesem Zusammenhang die Argumentation, mit der er sich im vierten Buch des Convivio gegen solch eine Behauptung vehement wehrt. Er tut dies dort just in einer Parenthese zu dem Abschnitt, der als »Erörterung gegen die Reichtümer» bekannt geworden ist. Thomas Ricklin hat hinsichtlich dieser Parenthese treffend geäußert, es sei Dante vermutlich daran gelegen gewesen, »den Wunsch nach

$21 »$ Veramente, al principale intendimento tornando, dico, come è toccato di sopra, per necessarie cagioni lo parlare di sé è conceduto: ed in tra l'altre necessarie cagioni due sono più manifeste. L'una è quando sanza ragionare di sé grande infamia o pericolo non si può cessare; e allora si concede, per la ragione che delli due sentieri prendere lo men reo è quasi prendere un buono.« (CV, I, ii, 12-14).

$22 »$ l'altra è quando, per ragionare di sé, grandissima utilitade ne segue altrui per via di dottrina $[. ..] \ll(\mathrm{CV}, \mathrm{I}, \mathrm{ii}, 14)$. 
Wissen [gezielt] als Alternative zum Wunsch nach Reichtum darzustellen. ${ }^{23}$ Dementsprechend vertritt unser Autor in der »disputazione [...] contro le ricchezze« (CV IV, XII, 11-XIII, 9) die These, Reichtum sei »niederträchtig«, und zwar vor allem, weil seine Anhäufung niemals Befriedigung schaffe, sondern im Gegenteil »im menschlichen Willen« ein unermessliches »Begehren« nach immer mehr erzeuge. Die Darlegung dieses Punktes unterbricht Dante sodann, um den grundsätzlichen Einwand zu entkräften, auch bei dem Verlangen nach Wissen handle es sich um ein Verlangen, das unersättlich sei und keine Grenzen kenne. Dem entgegnet er mit dem Argument, das Anwachsen von Wissen folge einem grundlegend anderen Prinzip als jenes von Reichtum: Während die »ricchezze« sich nämlich gewissermaßen ziellos vermehrten und daher unvollkommen blieben, strebe die "scienza» in vielen sukzessiven Schritten ihrer "perfezione« $\mathrm{zu}$ - und zwar einer Vollkommenheit, die der Mensch auch tatsächlich erlangen könne. Diese Vollkommenheit liegt für Dante in der Erkenntnis Gottes und das heißt in der schlussendlichen Rückkehr des Menschen zu seinem Ursprung. Die Erlangung dieses »höchsten Gutes« menschlichen Strebens verschafft dem Wissensdurstigen in seinen Augen daher jene endgültige Ruhe: »[C]hè nel desiderare de la scienza successivamente finiscono li desideri e viensi a perfezione, e in quello de la ricchezza no.« (CV IV; XIII, 5). Während also das Verlangen nach Wissenschaft durch das Erreichen von Vollkommenheit gestillt werden könne, gelte dies für das Anhäufen von Reichtum nicht.

In der Annahme, dass Dantes Verständnis von »scienza« das Ansammeln von an sich profanen Kenntnissen einschließt, lässt sich der hier skizzierte Gedankengang des Convivio als indirekte Legitimierung der Inszenierung des Ich in den Informationsgesprächen lesen, zumindest im Inferno und im Purgatorio. Denn das, was der wissensdurstige Dichter, der Fiktion zufolge, in ihnen über das sündig-lasterhafte Leben der Verstorbenen erfährt und in seinem poema festhält, steht unbestreitbar im Dienste der übergeordneten christlichen Heilsanthropologie - und somit im Zeichen der Rückkehr allen menschlichen Seins zu seinem Ursprung. Das in den Informationsgesprächen fortgesetzte Reden über sich selbst hat insofern vorderhand eine exemplarische Funktion, es ist der im Zusammenhang mit dem Paradiso erwähnten heilsgeschichtlichen Mission des Verfassers der Commedia zu subsumieren. ${ }^{24}$

23 Dante Alighieri, Das Gastmahl. Viertes Buch, Italienisch/Deutsch, übersetzt von Thomas Ricklin, eingeleitet und kommentiert von Ruedi Imbach in Zusammenarbeit mit Roland Béhar und Thomas Ricklin, Hamburg 2004, hier: »Inhaltsübersicht«, S. LXXVIII.

24 Siehe dazu eingehend Jörn Steigerwald, »Beatrices Lachen und Adams Zeichen: Dantes Begründung einer literarischen santhropologia christiana in der Divina Commedia (Paradiso IXXVII)«, in: Comparatio 3/2 (2011), S. 209-239. 
Problematisch daran bleibt allerdings, dass die heilsgeschichtliche Dimension in diesen Gesprächen ausgerechnet über das merkantile Argumentationsmuster des Tauschhandels vermittelt wird. Denn der dem Handel inhärente gegenseitige Verdienstgedanke, den die Dialoge über das Prinzip eines do ut des vermitteln, steht in deutlichem Widerspruch zum christlichen Heilsgedanken. Dieser bedarf, wie eingangs skizziert, gerade keinerlei Begründung oder argumentativer Herleitung, sondern beruht essentiell auf der Gnade Gottes. ${ }^{25}$ Im Ergebnis läuft dieser Befund darauf hinaus, dass die Commedia das Motiv des geistigen Handels bis zu einem gewissen Grad instrumentalisiert. Dies geschieht wesentlich im Zeichen der übergeordneten moraltheologischen Seinsordnung von Dantes christlichem Lehrgedicht.

\section{IV.}

Doch ist dies nur eine Seite der Medaille. Denn die Informationsgespräche zwischen >Dante und den Seelen verfolgen neben der dominant heilsgeschichtlichen Perspektive noch eine zweite Absicht. Diese weist in eine grundlegend andere Richtung und führt zu einer zweiten Lesart des merkantilen Argumentationsmusters, die von seiner ersten Deutung als einem exemplarischen Verfahren signifikant divergiert.

In seiner Verurteilung der selbstbezüglichen Rede im Gastmahl brandmarkt Dante besonders das Lob, das sich jemand selbst attestiert, als verderblich. Die Verwerflichkeit der trügerischen "propria loda" ist hier von Interesse, weil sie neben dem Sammeln des Stoffs für die Dichtung indirekt auf einen zweiten Nutzen verweist, den der Protagonist durch die Tauschgeschäfte mit den Seelen persönlich hat. Gemeint ist das Lob seiner Leistungen als Dichter. Auch dieser Profit bezieht sich unmittelbar auf die Figur `Dantes` und auch ihn thematisiert der Text meist lediglich diskret, und zwar durch die Zurschaustellung der Ehrerbietung, welche die Seelen dem poeta in den allermeisten Begegnungen wie selbstver-

25 Über die Gründe für diesen konzeptionellen Hiat zu spekulieren ist müßig. Doch scheint in diesem Punkt die kategoriale Differenz zwischen dem allegorisch kodierten Gedankensystem des Convivio und der Göttlichen Komödie, die jenes in eine Erzählung überführt, eine Rolle zu spielen. Dieser Diskurswechsel von einer theoretischen Darlegung zum Erzählen einer Geschichte nämlich bedingt den Einsatz spezifisch narrativer Verfahren, zu denen unter anderem die Dramatisierung des Gegenstands zählt. Im Zeichen einer solchen dramatischen Handlung scheint die Inszenierung der Tauschgeschäfte wesentlich zu stehen. Dies bedeutet, dass die Gestaltung der Dialoge nach dem merkantilen Argumentationsmuster vermutlich primär im literarischen Interesse der Commedia steht. 
ständlich erweisen. Die Ehrerbietung hat dabei in unserem Kontext wiederholt die Funktion einer Vorleistung, die diejenigen erbringen, die auf die Unterstützung des Dichters hoffen. Mit seltener Deutlichkeit bringt diesen Gedanken eine Stelle in Purgatorio V zum Ausdruck. ${ }^{26}$ In ihr klärt Vergil zwei Vertreter derjenigen, die gewaltsam aus dem Leben schieden, über die conditio desjenigen auf, dessen Körper die Sonnenstrahlen nicht durchlässt (Purg. V, 25f.) - gemeint ist selbstredend >Danteく. Die Belehrung hinsichtlich dieses lebendigen Menschen endet damit, dass Vergil die Neugierigen ermahnt, ihre »Leute« (V. 23) sollten Dante tunlichst Ehre erweisen, es könne ihnen gelohnt werden: »Faccianli onore, ed esser può lor caro.«(Purg. V, 36). Dem imperativen Tonfall dieses Aufrufs gehorcht die Schar der Angesprochen sogleich, indem sie Dante in einer Kollektivrede eilfertig fragen, ob er unter ihnen nicht vielleicht schon jemanden gesehen habe, von dem er »dort drüben « in der Welt Neuigkeiten verkünden wolle. ${ }^{27}$ Und unaufgefordert gestehen sie ihm, bis zur letzten Stunde Sünder gewesen zu sein, denen erst der gewaltsame Tod die Augen für das Licht des Himmels geöffnet habe. Dem Tauschgeschäft, das Dante in anderen Fällen seinerseits unterbreitet, kommen die "anime« in dieser Weise gewissermaßen zuvor und tragen ihren Teil dazu auch gleich aus eigenem Antrieb bei. Sie bringen dem Dichter wie selbstverständlich die Ehre entgegen, die Vergil eingefordert hat. Den zweiten Teil seiner Aussage, nämlich die Verheißung, dass der `Dante` entgegengebrachte »onore« zum Vorteil gereichen könne (»ed esser può lor caro«), löst das Ich sogleich seinerseits ein. Es fordert die Seelen auf, dass sie ihm sagen sollten, wenn sie einen Wunsch hätten, den er erfüllen könne, er werde es tun:

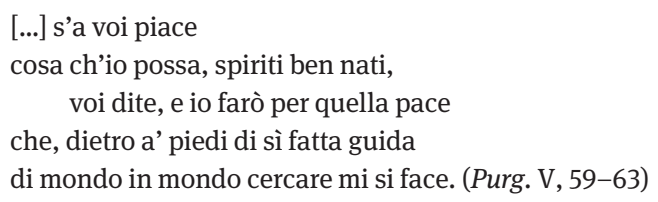

Von Bedeutung ist diese Sequenz, weil in ihr alle Ingredenzien des Tauschhandels gegeben sind, er dieses Mal jedoch von den Bewohnern des Antipurgatorio ausgeht, ohne dass die Regeln des »convegno « extra formuliert werden müssten. Dies scheint nicht nötig, da die Seelen offensichtlich wissen, was von ihnen erwartet wird, vor allem aber weil sie, Vergils autoritativer Aufforderung ent-

26 Siehe zu diesem Gesang der pentiti dell'ultima ora Michelangelo Picone: „Canto V«, in: Lectura Dantis Turicensis. Purgatorio, a cura di G. Güntert e M. Picone (2001), S. 71-83 sowie Pasquale Guaragnella: »Fede cristiana e pentimento. Due storie della `Commedia` di Dante«, in: Deutsches Dante-Jahrbuch 85/86 (2010-2011), S. 73-86.

27 »Guarda s'alcun di noi unqua vedesti, / sì che di lui là novella porti: [...]« (Purg. V, 49-50). 
sprechend, dem Dichter ihre Ehre erweisen. ${ }^{28}$ Die Ehrerbietung äußert sich dabei vor allem in einem uneingeschränkten Vertrauen, das die Jenseitigen in Dante setzen: das Vertrauen darauf, dass dieser alles in menschlicher Macht stehende tun wird für jene, die ihm Ehre bezeugen. Genau diese Überzeugung repräsentiert in demselben Passus eine Stimme, die aus der Menge der Seelen hervortritt und >Dante` gegenüber feierlich im Namen aller anwesenden Seelen beteuert:

\section{[...] $»$ Ciascun si fida}

del beneficio tuo senza giurarlo, pur che 'l voler nonpossa non ricida.« (Purg. V, 64-66) $)^{29}$

In den bisherigen Beispielen wurde die Bürgschaft, die der Dichter in der Übereinkunft übernimmt, indem er potentiell für einen moralisch verlässlichen Nachruf der Seelen in der Welt eintritt, als eine Leistung gekennzeichnet, der eine Gegenleistung entspricht. In der ehrfürchtigen Haltung der Seelen in Purgatorio V hingegen tritt an die Stelle des beiderseitigen do ut des auf Seite der Seelen gewissermaßen die vertrauensvolle, vorauseilende Dankbarkeit gegenüber dem Dichter der Commedia. Von einer Gegenleistung >Dantes` für das ihm entgegengebrachte Ehrerbieten kann recht besehen keine Rede sein, denn sein Angebot, sich für ihr Seelenheil einzusetzen, folgt dem Gestus einer souveränen Wohltat (»beneficio«, V. 65), anstatt eine Vereinbarung zu sein.

Die hier zu beobachtende, scheinbar marginale Verschiebung weg vom Prinzip des gegenseitigen Gebens hin zu einem einseitig formulierten Vertrauensverhältnis ist bedeutsam, denn sie reduziert den geistigen Handel auf ein bedingungsfreies Subordinationsverhältnis, bei dem einer gibt und der andere nimmt. Dabei vertrauen die Seelen auf `Dante`als eine wirkungsmächtige Instanz, die wohltätig für ihre Interessen eintreten wird. Auf diese Weise aber erweisen sie ihm jene Form von Ehre, die zu Dantes Zeit einer Autorität zugeschrieben wurde. Damit ist gemeint, dass der fünfte Gesang des Purgatorio ein hierarchisches Vertrauensverhältnis ins Bild setzt, das ziemlich genau jener Art von Hochachtung entspricht, die man nach

28 Siehe zum Themenkomplex des onore, das im Purgatorio eine zentrale Position im Spannungsfeld von humilitas und superbia einnimmt, Paolo Marini, » $)$ La gloria de la lingua nel trittico dei superbi. Considerazioni sul nodo arte-onore-superbia-umiltà nella `Commedia«", in: Italianistica 36 (2007), 65-88 sowie Michael Schwarze, »Lectura Dantis: Purgatorio XI«, in: Deutsches DanteJahrbuch 85/86 (2010-2011), S. 237-269.

29 Chiavacci Leonardi (wie Anm. 9, vol. 2: Purgatorio, Milano 1994, S. 147) paraphrasiert den schwierigen Vers 66 wie folgt: "purché l'impossibilità, il non potere (nonpossa) non tronchi, non interrompa il tuo volere (cioè purché tu abbia la possibilità materiale di farlo, visto che il volere certamente lo avrai).« 
zeitgenössischen Vorstellungen einer auctoritas zu zollen hatte. ${ }^{30}$ Der semantische Kern, der sich seit der Antike mit der Vorstellung von Autorität verband, bestand in der Auffassung, die auctoritas einer Person basiere darauf, dass sie in ihrem Handeln als Garant für eine Sache einstehe und daher uneingeschränktes Vertrauen verdiene. In seiner abstrakten Verwendung bezog sich der Begriff in aller Regel auf bestimmte Texte antiker oder christlicher Autoren, denen eine zeitlose Exemplarität beigemessen wurde und die daher unantastbare Glaubwürdigkeit besaßen. Dieser Grundannahme folgt auch die etymologische Herleitung der »filosofica autoritade«, die Dante im Convivio vornimmt. Mit Bezug auf Aristoteles versteht er unter Autorität dort im vierten Buch, kurz gesagt, die »Handlung eines Autors« (»atto d'autore«), die »des Vertrauens und des Gehorsams würdig ist«:

E così, sautore [ [...] si prende per ogni persona degna d'essere creduta e obedita. E da questo viene questo vocabulo [...], cioè rautoritade‘; per che si può vedere che rautoritade` vale tanto quanto satto degno di fede e d'obedienzar. (CV IV; VI, 5)

Diese Definition lässt sich recht umstandslos auf die behandelte Handlungssequenz in Purgatorio V zurückprojizieren. Vor ihrer Folie erscheint die dort inszenierte Ehrerbietung gegenüber der Figur des heilbringenden >Dante als eine versteckte Erhebung des Dichters in den Status einer Autorität. Dies aber bedeutet nichts anderes, als dass der Text der Commedia das Gespräch gezielt auch in der Absicht inszeniert, das Ich mit einer Qualität auszustatten, welche es insgeheim für sich beansprucht, jedoch nicht offen formulieren kann.

Mit dem Verhältnis von Autorschaft und Autorität in der Commedia ist ein Problemfeld angesprochen, das seit jüngerer Zeit die Danteforschung beschäftigt. Neben den Arbeiten von Gerhard Regn ${ }^{31}$ ist in diesem Zusammenhang vor allem auf die 2011 in zweiter Auflage erschienene Studie Dante and the Making of a Modern Author hinzuweisen, in der Albert Russell Ascoli diese Zusammenhänge aufgearbeitet und magistral neu perspektiviert hat. ${ }^{32}$ Sie zeigt, wie, auf welchen

30 Siehe zum Folgenden prägnant Giorgio Stabile, »autorità (AUTORITADE)«, in: Enciclopedia Dantesca, vol. 1, S. 456-460.

31 G. Regn, "Double Authorship« (wie Anm. 18) und ders., "Gott als Dichter« (wie Anm. 18). Siehe zu Entwicklungslinien des Phänomens autoritativer Selbstkonstruktion ausgehend von Dante und mit Blick auf die humanistische Liebesdichtung David Nelting, »Frühneuzeitliche Selbstautorisierung zwischen Singularisierung und Sodalisierung (Francesco Petrarca, Pietro Bembo, Joachim Du Bellay)«, in: Romanistisches Jahrbuch 62 (2011), S. 188-214.

32 Albert Russel Ascoli, Dante and the Making of a Modern Author, Cambridge University Press ${ }^{2} 2011$. Von Ascoli stammt auch die Charakterisierung von Inferno I, 85-87 als translatio auctoritatis; vgl. ebd., S. 357. 
Grundlagen und mit welchen Zielen der Verfasser der Commedia vom Moment der ersten Begegnung mit Vergil im Inferno und der damit einhergehenden translatio auctoritatis bis zu den letzten Gesängen des Paradiso das eigene Ich als einen »autore« konstruiert, dessen Autorität ihresgleichen sucht. Ausgehend von der Modellierung des Tauschhandels im fünften Purgatoriumsgesang scheint mir an der Selbstkonstruktion des Dichters dabei ein Detailaspekt besondere Aufmerksamkeit zu verdienen: Er betrifft die Frage, wie man zu einer Autorität wird, oder präziser gesagt, wer einen in diesen Status erheben kann. Soweit ich sehe, hat unser Autor diese Frage nach dem Modus der Autoritätsstiftung selbst nicht ausdrücklich thematisiert. Die Weise jedoch, in der er an besagter Stelle und andernorts in der Commedia die Ehrerbietung der Seelen gegenüber dem Ich in Szene setzt, weist mittelbar auf dieses Problem hin. Es drängt sich dabei der Eindruck auf, der Text delegiere in solchen Momenten das unmögliche Eigenlob, das in der Selbstzuschreibung ehrwürdiger Autorität läge, gewissermaßen an die Gesprächspartner >Dantes`. Die ehrerbietende Haltung der Seelen bezeugt somit gewissermaßen in actu eine Dichterautorität, die insgeheim als gegeben supponiert wird und die der Dichter selbst bezeichnenderweise an keiner Stelle der Commedia für sich reklamiert. ${ }^{33}$ Auf diese Weise aber markiert der Text der Commedia de facto stets aufs Neue, dass er sich jene "autoritade« eigentlich nicht selbst attribuieren darf. Der Grund dafür ist über das Argument des Selbstlobs hinaus, dass es sich bei der Autorität um eine Qualität handelt, die einem Text (und im Weiteren dem Autor) erst auf dem Wege seiner Rezeption und das heißt von Dritten zugeschrieben werden kann. ${ }^{34}$ Genau diese Funktion aber haben in den behandelten Dialogen >Dantes` Gesprächspartner inne. In diesem Lichte betrachtet erweist sich das einseitig formulierte Vertrauensverhältnis, das Dante in Purgatorio V evoziert, nicht zuletzt als Teil einer weiterreichenden Textstrategie, die im Zeichen der heiklen Selbstkonstruktion des Ich zu einer literarischen Autorität steht.

33 Ascoli (wie Anm. 32), S. 9 führt für das Gesamtwerk Dantes nur zwei Stellen an, in denen der Dichter die Wörter »Autorität« oder »Autor« in Bezug auf sich selbst verwendet: CV I.iv.13 und Ep. 3.2-3.

34 Das rezeptive Konstituens von Autorität kommt bei Dante am ehesten darin zum Ausdruck, dass er im Convivio, wie gesehen, betont, eine Autorität müsse des Vertrauens und des Gehorsams »degno« sein. Die Auffassung, dass sich Autorität allererst in der Rezeption eines Textes ausbildet, wird durch den Blick auf die historische Praxis der Autoritätsstiftung im Mittelalter bestätigt: Bei den auctoritates handelte es sich stets um antike oder christliche Autoren der Vergangenheit, denen erst nachfolgende Generationen von Denkern post mortem eine zeitlose Bedeutung attestieren konnten. Siehe dazu grundlegend Hans Robert Curtius, Europäische Literatur und lateinisches Mittelalter, Tübingen ${ }^{11} 1993$, S. 58-70. 
Die Untersuchung der Informationsgespräche, die der Jenseitspilger Dante vor allem mit den Seelen in der Hölle und auf dem Läuterungsberg führt, hat gezeigt, dass diese vielfach Züge eines geistigen Tauschgeschäfts aufweisen. Sie folgen in der Regel dem Grundsatz des gegenseitigen Nutzens, den der Dichter womöglich vor allem deshalb so nachdrücklich inszeniert hat, weil er narrativen Erfordernissen seiner Geschichte gerecht $\mathrm{zu}$ werden suchte. Eine Folge dieser Dramatisierung des Stoffes ist, dass das Argumentationsmuster des do ut des in diesen Gesprächen das ihm übergeordnete christliche Deutungsmuster in konzeptioneller Hinsicht kontaminiert. Vor allem aber lässt sich beobachten, dass der Motivkomplex des geistigen Handels in der Commedia von einer grundlegenden Ambivalenz gekennzeichnet ist. Diese liegt darin begründet, dass die Tauschgeschäfte hier gleichsam als Diener zweier Herren fungieren: Sie stehen zugleich im Zeichen der exemplarischen Belehrung wie der selbstkonstruktiven Funktion des Dichters. Diesen Befund kann man als einen ungewollten gedanklichen Hiat begreifen, der sich im Kontext der Commedia kaum auflösen lässt. Man kann ihn aber auch, wie hier geschehen, als Ausdruck einer gezielten textuellen Strategie verstehen, die bewusst eine ambiguisierende Wirkung erzeugen will und daher diskrepante Deutungen insinuiert. Diese Strategie ließe sich pointiert dahingehend zuspitzen, dass die geistigen Abkommen in der Commedia gezielt zugleich als Geschäfte im Namen Gottes und im Namen eines Autors auf der Suche nach Autorität inszeniert werden. Den plausiblen Ausgangspunkt für eine solche Lesart stellt die erwähnte zweifache Mission dar, die dem Ich in Dantes Darstellung von Beginn an attribuiert wird. Denn indem die Ich-Figur gleichermaßen als Christenmensch angelegt ist, der die Erlösungstat Christi quasi ein zweites Mal vollbringt, und als Verfasser der Commedia, die davon ein einmaliges literarisches Zeugnis ablegt, schreibt sich der Text eine Autorität zu, die durch ihre Exemplarität gerechtfertigt, hinsichtlich ihrer Selbstbezüglichkeit jedoch äußerst prekär ist. Im Zeichen dieses Projekts, in dem heilsgeschichtliche und mundane Einzigartigkeit gezielt amalgamiert werden, steht meines Erachtens eine Ambiguisierungsstrategie der Darstellung, welche sich in dem hier untersuchten Do-utdes-Muster prägnant verdichtet.

Es gehört zur Einzigartigkeit Dantes, dass die Commedia schon sehr früh nach seinem Tode in unzähligen Handschriften zirkulierte, welche mit einer rapide anwachsenden Zahl von lateinischen und italienischen Kommentaren versehen waren. Seiner auf Italienisch geschriebenen Dichtung wurde damit bereits in der zeitgenössischen Rezeption eine Verbreitung und Deutungsdichte zuteil, die im Bereich des volkssprachlichen Schrifttums des Spätmittelalters singulär ist. Dantes christliches Epos avancierte so binnen kürzester Zeit tatsächlich zu einer unumschränkten Autorität, die Gelehrte und Laien mit dem gleichen Respekt auslegten, wie sie dies seit jeher mit der Bibel und den `klassischen 
taten. Angesichts dieses rasanten Aufstiegs in den Rang einer gleichsam >göttlichen Autorität‘ scheint es, als sei Dantes Doppelstrategie aufgegangen. Tatsächlich ist für diese Apotheose jedoch vor allem eine Reihe sozialer, politischer, aber auch ökonomischer Transformationsprozesse verantwortlich, deren Zusammenspiel zumal in Dantes Heimatstadt Florenz eine mächtige Laienkultur schuf. »Geist und Geld « spielten dabei vielfältige Rollen, deren Untersuchung anderen Annäherungen an das Thema überlassen bleiben muss. 